\title{
Incorporación masiva de la mujer al mundo laboral,factor determinante en las políticas de armonización Trabajo - Familia ${ }^{1}$
}

\author{
Las tendencias actuales demuestran la creciente importancia \\ de las políticas relativas al trabajo y a la familia, que hacen \\ necesario lograr un equilibrio entre las cuestiones laborales $y$ \\ ese núcleo fundamental de la sociedad. \\ BAN Ki - moon Secretario General de Naciones Unidas \\ Adria Velia González Beltrones ** \\ Guadalupe Aleida Valenzuela Miranda* \\ María Dolores Rocha Ontiveros* \\ Alejandra Romano Nuñez
}

\section{Sumario: Introducción II.Algunos supuestos III. Algunos Datos estadísticosy sus gáficos en México IV. Agentes implicados V.Conclusiones}

\section{Resumen}

Se destaca la conveniencia de valorar en su verdadera dimensión el fénómeno sociodemográfico de la incorporación desde finales del siglo pasado de la mujer en forma masiva al mundo laboral, lo que impacta directamente los espacios Familia - Trabajo.Mujeres y hombres integran,participan,forman e interactuan a la vez en el mundo del trabajo y el de la familia.Distintos agentes sociales como el Estado,las administraciones públicas intervienen en su favor atraves de la expedición de leyes de seguridad social,laboral etc,que indiscutiblemente deben ser observadas por los empleadores tanto públicos como privados. Por lo que cabe cuestionarse en que medida, conviene elaborar políticas complementrias desde las empresas a favor de los y las trabajadoras,que favorezcan la armonía entre los ámbitos Trabajo -Familia ---como por ejemplo horario laboral flexible, jornada laboral reducida,empleos compartidos, prestaciones paternidad/maternidad,estancias infantiles,becas escolares entre otras---,de manera tal que su implementación sea valorada por la planta laboral en el mismo plano que se valora la retribución salarial al mismo tiempo que la empresa se integra al numero de empresas socialmente responsables y coadyuvan a la consecución de los Objetivos del Milenio(ODM)

Palabras clave:familia,conciliación,trabajo

\footnotetext{
1 Ponencia aceptada para su presentación en el XII Coloquio Nacional de la Red de Estudios de Género del Pacífico Mexicano Uiversidad Autónoma de Nayarit UAN,Junta Local Ejecutiva del INE Nayarit Nuevo Vallarta,Nayarit 10.11 y 12 de junio de 2015.Mesa de Trabajo1. Armonización de la vida laboral y familiar

- Docentes Investigadoras del Departamento de Derecho de la Universidad de Sonora integrantes del CAEC 109 de la Universidad de Sonora

** Estudiante Becaria Ayudante de investigador adscrita al CAEC 109 USON
} 


\begin{abstract}
This paper highlights the desirability of valuate in its true dimension the sociodemographic phenomenon of the massively incorporation --since the end of the last century-from the woman to the working world, which directly impacts the family - work spaces.Men and women make up, participate, integrate and interact at the same time in the world of work and the family.Different social agents as the State, the public administrations involved in his favor through the issuing of laws of social security, employment etc, which arguably should be observed by both public and private employers.
\end{abstract}

Therefore, we should to question in what measured should the companies be to elaborate complementary policies in favor of workers in order to benefit the harmony between work and familiy áreas --for example schema work flexible, reduced working hours, shared jobs, paternity/maternity benefits, childcare, scholarships among others--,in such a way that its implementation be valuated by the workforce in the same plane that is valuated the wage compensation, at the same time that the company is integrated to the number of socially responsible companies and both contribute to the achievement of the Millennium Goals (MGs) Key words:Family, work conciliation

\title{
Introducción
}

Tradicionalmente la "división sexual del trabajo" asigna a las mujeres la mayor parte del trabajo no remunerado en el hogar, incluyendo el cuidado de adultos mayores con o sin menoscabo de sus facultades. De esta división sexual del trabajo se derivan una "serie de discriminaciones y desigualdades, muchas veces invisibilazada, que impiden la autonomía económica de las mujeres, limitan sus derechos y merman sus libertades ${ }^{2}$

En relación a esta realidad, los organismos internacionales como la ONU,OEA han puesto en el tapete de la discusión de la mayoría de los países miembros la necesidad de erradicar todas las formas de discriminación contra la mujer y por

\footnotetext{
2 Relatoría Novena Reunión Internacional "Políticas públicas, uso del tiempo y economía del cuidado: la importancia de las estadísticas nacionales
} 
ello solicitan informes en relación a la observancia de los $D D H H$ de hombres y mujeres en general y en específico de la mujer y las políticas públicas al respecto. La respuesta de la mayoría de los países muestran los avances asimétricos y la urgente necesidad de modificar esta situación a través de políticas públicas que a su vez sumen al sector privado en el objetivo de alcanzar una sociedad más igualitaria.

Al efecto, en algunos países se han llevado a cabo investigaciones teóricas y empíricas para cuantificar y valorar la contribución total del trabajo de las mujeres, al desarrollo y se han dado pasos legislativos para colocar en las agendas públicas la medición del trabajo no remunerado así como la promoción de políticas públicas sobre la economía del cuidado (Ecuador, Colombia, Perú, Bolivia, Costa Rica y México entre otros ).

En ésta línea, ONU Mujeres, INEGI, la CEPAL y el INMUJERES organizaron la IX Reunión Internacional "Políticas Públicas, Uso del Tiempo y Economía del Cuidado: la importancia de las estadísticas nacionales" ${ }^{3}$, cuyo objetivo general era "impulsar el intercambio, la reflexión y la difusión de experiencias en materia de gestión de información, conocimientos y políticas públicas sobre uso del tiempo y economía del cuidado basadas en evidencias empíricas. ${ }^{4}$ Los objetivos especificos se centraron en la discusión en torno a los instrumentos de recolección de información sobre uso del tiempo y trabajo no remunerado, metodologías, alcances y limitaciones de la información -tanto conceptuales como operativos- y compartir las experiencias en el cálculo de las cuentas satélite del trabajo no remunerado en la región. $Y$ en conocer y compartir las experiencias en torno a los avances en las legislaciones en materia de trabajo no remunerado de cuidados en la región."

\section{Planteamiento del problema}

En este contexto, en relación al tema que nos ocupa, retomaremos lo relacionado

\footnotetext{
${ }^{3}$ la cual tuvo lugar el 29 y 30 de agosto de 2011 en la Ciudad de México

${ }^{4}$ Ibid pp2
} 
con los resultados de la Cuenta Satélite del Trabajo no Remunerado de los Hogares de México (CSTNRHM) que tiene el propósito de dimensionar de manera más precisa el aporte de los hogares a la economía nacional, al presentar la producción y el consumo de los servicios del hogar no medidos en la contabilidad nacional. ${ }^{5}$ Y que tal vez ofrezca una luz para orientar los esfuerzos de los ámbitos público y privado, para resolver la problemática que implica la relación de los espacios Trabajo-Familia según demuestran diversos estudios como el de Alderfer $^{6}$ que relaciona hábitos operativos y modos de relación familiar, demuestran que en muchos casos algunos modos de relación y organización vivídos en la familia pueden proyectarse en la empresa.

Por lo que cabe cuestionarse ¿en que medida desde las orgnizaciones públicas y/o privadas--- en nuestro entorno-se diseñan políticas complementarias a las derivadas del cumplimiento de obligaciones en materia laboral y de seguridad social, que favorezcan la armonía entre los espacios Trabajo/Familia? ${ }^{7}$

\footnotetext{
${ }^{5}$ INEGI,PIB y Cuentas Nacionales de México 2013.[online]Disponible en:http://www.inegi.org.mx/est/contenidos/proyectos/cn/tnrh/

${ }^{6}$ Alderfer,Charleen,1994,A response to a feminist critique of organizational humanism, Journal of applied Behavioral Science,Vol.30
}

7 ---tales como flexibilidad en los horarios,jornadas laborales compartidas, licencia por paternidad o maternidad, entre otras--,cuyos efectos positivos se proyecten en el trabajo de manera tal que se valore por el o la trabajador(a)como una fuente de retribución laboral tan importante como la salarial y que al empleador le facilite formar parte del grupo de empresas socialmente responsables coadyuvantes en la consecución de los Objetivos de desarrollo del Milenio ODM Los Objetivos de desarrollo del Milenio:

- Consolidan muchos de los compromisos más importantes asumidos por separado en las cumbres y conferencias de las Naciones Unidas en la década de los 90;

- Reconocen explícitamente la dependencia recíproca entre el crecimiento, la reducción de la pobreza y el desarrollo sostenible;

- Consideran que el desarrollo se sustenta en la gobernabilidad democrática, el estado de derecho, el respeto de los derechos humanos, la paz y la seguridad;

- Están basados en metas cuantificables con plazos y con indicadores para supervisar los progresos obtenidos; y

- Combinan, en el octavo Objetivo, las responsabilidades de los países en desarrollo con las de los países desarrollados, sobre la base de una alianza mundial respaldada en la Conferencia Internacional sobre la Financiación para el Desarrollo celebrada en Monterrey, México, en 2002 y reafirmada en la Cumbre Mundial sobre Desarrollo Sostenible celebrada en Johannesburgo en agosto de 2002. ONU México,[online]Disponible en: http://www.onu.org.mx/objetivos_de_desarrollo_del_milenio.html ${ }^{\wedge}$ consultado el 12/05/2015 


\section{Algunos supuestos:}

En México hay ausencia de políticas públicas y de andamiaje normativo, que favorezcan la armonía entre los espacios Trabajo/Familia, complementarias a las derivadas del cumplimiento de obligaciones en materia laboral y de seguridad social.

Las empresas del sector privado en México no visualizan la importancia de contar con programas trabajo/familia que repercutan en el aumento de productividad, y el abatimiento del absentismo, y de la rotación de empleados.

De los resultados de la reunión internacional precitada nos interesa lo relacionado especialmente con la estadística nacional con perspectiva de género puesto que ello ayuda a dimensionar las desventajas y las diferencias de género en los ámbitos público y privado.

Así, en palabras de Eduardo Sojo Garza - Aldape ${ }^{8}$ "en los últimos años se ha visto claramente que las estadísticas nacionales son indispensables para las políticas públicas y ayudan a transformar las agendas sectoriales, asimismo ...existe la necesidad de prestarle más atención a la igualdad y en dar una mayor visibilidad al papel económico, político y social que día con día desempeñan las mujeres $^{9} \ldots . .$.

Por su parte, Dolores de los Ángeles Nazares ${ }^{10}$ considera ... que la ejecución de políticas públicas en general, y en particular las dirigidas a la atención de las mujeres,......requieren un sustento cuantitativo y cualitativo robustos que den plena

\footnotetext{
${ }^{8}$ Presidente del Instituto Nacional de Estadística y Geografía (INEGI) de México (2011) y Coordinador del Grupo de Trabajo sobre Estadísticas de Género de la Conferencia Estadística de las Américas (CEA) de la CEPAL;

${ }^{9}$ enfatizando el relevante papel que debe jugar el Comité de Información con Perspectiva de Género para garantizar que todos los proyectos estadísticos de las instituciones que conforman el Sistema Nacional de Información Estadística y Geográfica (SNIEG) incorporen la visión de género.

Dolores de los Ángeles Nazares, Presidenta de la Comisión de Equidad y Género (2011)en la Cámara de Diputados de México.

${ }^{10}$ Presidenta de la Comisión de Equidad y Género (2011)en la Cámara de Diputados de México.
} 
certeza a los formuladores y ejecutores de acciones y programas, sobre los objetivos que se pretenden alcanzar.

En el mismo tenor Luis Beccaria y Sonia Montaño ${ }^{11}$ señalan que ya son varios los países que han realizado algún levantamiento y que cuentan con información, y que ......las encuestas de uso del tiempo dan cuenta de la discriminación que ejercen las sociedades hacia las mujeres. En igual línea, Rocío García Gaytán ${ }^{12}$ señala que la Cuenta Satélite del Trabajo no Remunerado de los Hogares de México CSTNRHM representa un avance sin precedentes en la producción de insumos para el diseño de políticas públicas dirigidas a la igualdad entre mujeres y hombres (ver gráficos). Que hay dos acciones de gobierno determinantes, la primera que el presupuesto para la igualdad ha logrado un incremento constante; la segunda que la reforma de la Ley de Planeación requiere la generación de indicadores para medir el impacto de los programas gubernamentales en mujeres y hombres.

De la presentación de la CSTNRHM (2013), La Medición de la economía del cuidado, ${ }^{13}$ tenemose

\section{Algunos datos estadísticos y sus gráficos en México}

México cuenta con : Dos levantamientos de la Encuesta Nacional de Uso del Tiempo, con información referida a los años de 2002 y 2009;los trabajos de la Valoración económica del trabajo no remunerado comenzaron en el año 2009;

La primera difusión de resultados fue en agosto del 2011 mediante una serie de tiempo del 2003 al 2009, presentando: Métodos de valoración, valoraciones en cifras netas y cifras brutas y estratos poblacionales.

A partir del 2011 se publica anualmente la Cuenta satélite del trabajo no remunerado de los hogares.

11 En 2011 Director de la División de Estadística y Proyecciones Económicas de la CEPAL de Chile; y Directora de la División de Asuntos de Género de la Comisión de Estadística para América Latina (CEPAL) de Chile respectivamente.

${ }^{12}$ Presidenta de Instituto Nacional de las Mujeres (INMUJERES) de México (2011);

${ }^{13}$ INEGI,Conociendo México,2013[online]Disponible en :

https://www.dane.gov.co/files/noticias/eventos/inegi_rmlg.pdf 
En 2012, se actualizó el cálculo al año 2010 y se agregaron dos estratos poblacionales: Quintil del hogar y Condición de habla indígena.

En mayo 2013, Actualización al año 2011 y se publicó el Valor del trabajo no remunerado en la producción de bienes de autoconsumo.

En agosto 2013, Actualización del año base 2008 para la serie del 2003 al 2011.

La cobertura del Trabajo no remunerado de los hogares es el "Tiempo utilizado en las labores domésticas y los cuidados realizados por los miembros del hogar para producir servicios destinados al consumo del hogar, sin obtener un pago o remuneración, y que estén fuera de la frontera de la producción de la contabilidad nacional."

Labores domésticas y de cuidados = Economía del Cuidado

Una actividad es productiva si se puede delegar a una tercera persona, si proporciona un producto o servicio que se puede intercambiar.(Proporcionar alimentos, proporcionar limpieza y mantenimiento a la vivienda,proprcionar limpieza y cuidado de la ropa y calzado, proporcionar compras y administración del hogar, proporcionar cuidados y apoyo, proporcionar ayuda a otros hogares y trabajo voluntario

El trabajo no remunerado de los hogares se realiza: En el propio hogar, para otros hogares, para la comunidad y trabajo voluntario. 
BIOLEX Revista Jurídica del Departamento de Derecho UNISON URC

Academia de Derecho Administrativo

Tercera Época Año 7. No 12 Enero-Junio de 2015.

\section{Trabajo no Remunerado de los Hogares}

Simuladlor $\equiv \underline{\text { Más informaciól }}$

Valor económico de las labores domésticas y de cuidados no remunerados

Porcentaje y millones de pesos

\begin{tabular}{lr}
\multicolumn{1}{c}{ Concepto } & $2013^{\mathrm{P}}$ \\
\hline Valor respecto del PIB nacional a precios de mercado & $20.5 \%$ \\
Valor del trabajo no remunerado en labores domesticas y de cuidados & $3,302,737$ \\
Alimentación & $23.3 \%$ \\
Limpieza y mantenimiento de la vivienda & $15.6 \%$ \\
Limpieza y cuidado de la ropa y calzado & $8.5 \%$ \\
Compras y administración del hogar & $12.9 \%$ \\
Cuidados y apoyo & $34.6 \%$ \\
Ayuda a otros hogares y trabajo voluntario & $5.1 \%$
\end{tabular}

${ }^{\mathrm{P}}$ Cifras preliminares

Fuente: INEGI Conociendo México,2013[

Valor anual de las labores domésticas y de cuidados no remuneradas por persona que lo realiza, $2013^{P}$

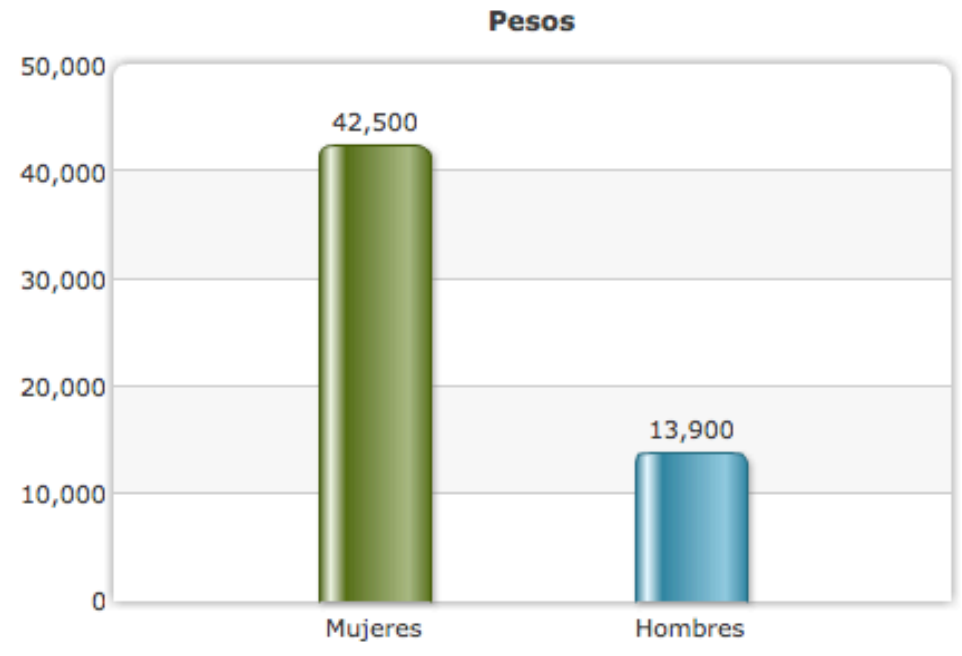

${ }^{\mathrm{P}}$ Cifras preliminares 
BIOLEX Revista Jurídica del Departamento de Derecho UNISON URC

Academia de Derecho Administrativo

Tercera Época Año 7. No 12 Enero-Junio de 2015.

Participación de las labores domésticas y de cuidados no remuneradas y de algunos sectores económicos respecto del PIB, 2013 ${ }^{\mathrm{P}}$

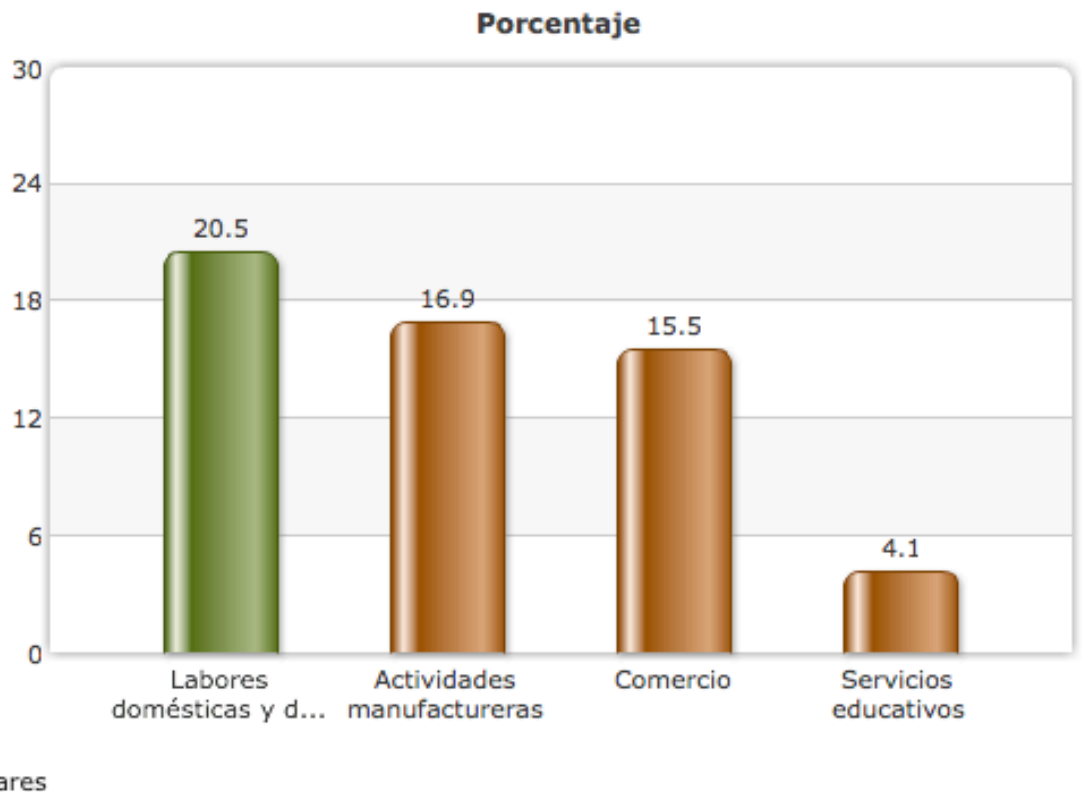

${ }^{\mathrm{P}}$ Cifras preliminares

\section{Fuente INEGI:Conociendo México,2013}

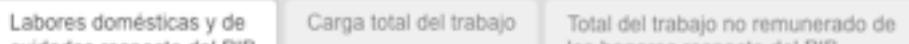

Labores domésticas y de cuidados respecto del PIB

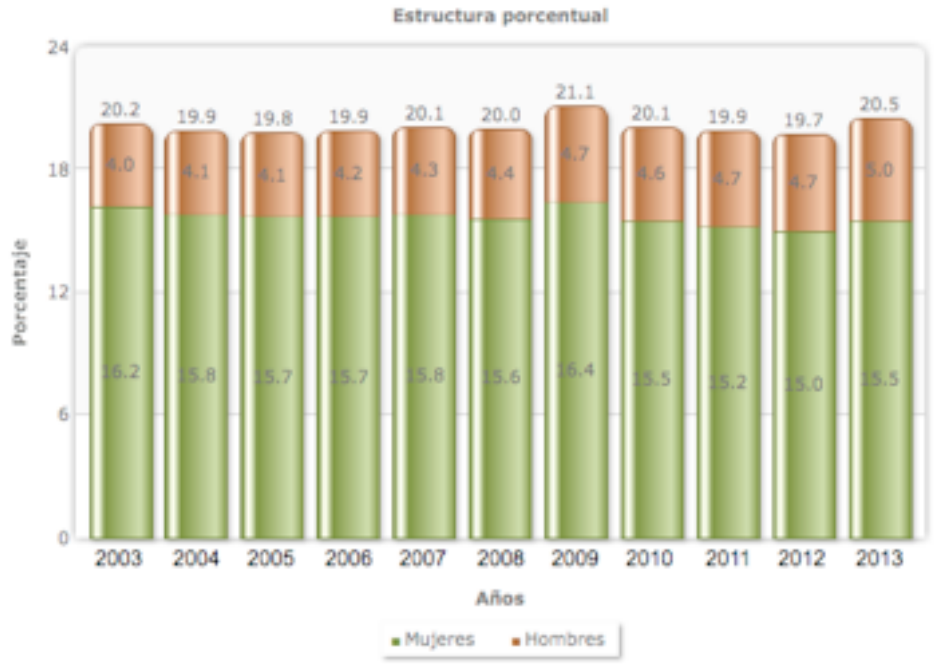

Nota: cifras preliminares a partir de 2012

La suma de los parciales puede no coincidir con el total debido al redondeo. 
Resultados : El valor de las labores domésticas y de cuidado no remuneradas resultó mayor a algunas actividades económicas del país(2013) :

\section{5 veces mayor que las actividades manufactureras}

5.0 veces mayor que el comercio

16.4 veces mayor que los servicios educativos

Las mujeres realizan las labores domésticas y de cuidados no remunerados en un porcentaje $5.5 \%$ mayor que los hombres representando un valor anual de $\$ 42,400.00$ aproximadamente cuatro veces mas que el aportado por los hombres $\$ 13,900.00$.

Los datos anteriores hacen visible las muchas prácticas discriminatorias hacia las mujeres trabajadoras y/o hacia las dedicadas exclusivamente a las labores del hogar, que aún imperan en el país, por la falta de valoración y reconocimiento de su participación en la economía del cuidado nacional no medido en la contabilidad nacional, por lo que urge institucionalizar en leyes laborales y fiscales las políticas públicas que promueven la armonización de los ámbitos laboral y familiar y buscan el objetivo de abatir las desigualdades que existen al interior del hogar y sus lamentables efectos sobre las mujeres

\section{Agentes implicados}

En este contexto, es importante abordar separadamente la intervención de los agentes implicados

\section{a)Sector público}

Ante la urgente necesidad de prestarle más atención a la igualdad y en dar una mayor visibilidad al papel económico, político y social que día con día desempeñan las mujeres en México ${ }^{14}$, se requiere la intervención del ente público

14 si bien se ha incrementado de manera notable, también es cierto que se da en condiciones desventajosas: mayores cargas de trabajo(por la suma de trabajo remunerado y no remunerado), lo cual restringe sus oportunidades, limita sus expectativas y libertades para realizar actividades 
competente en la elaboración de diagnósticos por entidad federativa: sobre el trabajo remunerado y no remunerado en el hogar y fuera del hogar, sobre el cuidado de menores y/o mayores discapacitados y sus repercusiones en la sobrecarga y en la inequitativa distribución de responsabilidades a hombres y mujeres.

Una vez realizados los diagnósticos respectivos, darán sustento cuantitativo y cualitativo al diseño de políticas públicas, institucionalizadas en leyes, acciones y programas que persigan el objetivo de abatir las desigualdades y descargar a las familias y a las mujeres especialmente de asumir en solitario la responsabilidad de armonizar el trabajo y la familia.

\section{b)Sector privado}

Dado que la sociedad de nuestros días está cambiando su perfil ${ }^{15}$, la familia concebida tradicionalmente como unidad y espacio en el que el cuidado material del hogar y de los hijos le corresponde a la mujer, hoy en día, éste rol es compartido entre cónyuges dado que también se comparte: el solventar el gasto familiar ---con lo obtenido por su desempeño como profesionales en el mundo laboral --y las actividades relacionadas con la educación de los hijos.

Esta cambiante realidad, trasciende al hogar y provoca que muchas personas vivan un conflicto entre el trabajo y la familia lo que repercute directamente en el ámbito laboral y viceversa: Las noveles parejas, retrasan nacimientos o deciden no procrearlos con tal de conseguir ascensos o encontrar o conservar un empleo -- demandante a veces, de tiempos extras o de constantes desplazamientos dentro y fuera del país---, experimentan dificultades de toda índole de acceso a vivienda y a automóvil, sobrellevan la inestabilidad en el empleo, entre otros

remuneradas, lo que a su vez contribuye a robustecer la desigualdad en otras esferas de la vida de las mujeres.

15 Descensos bruscos de la natalidad, aumento de familias monoparentales,---por divorcios o concubinatos o sin estructura institucional—etc. 
aspectos; a su vez, la inflexibilidad de las empresas en los horarios de entrada --sin tomar en cuenta la carencia de transportación pública urbana efectiva ---la falta de estancias infantiles o de adultos mayores, y lo de escuelas --- accesibles y dentro de la ruta al trabajo_provocan en las parejas y/o en la familia, estrés al tratar de combinar trabajo y familia.

La mayoría de las empresas consideran que el conflicto entre el trabajo y la familia no es de su responsabilidad. Los empleados a su vez esperan que los gobiernos y los patrones lo resuelvan.

\section{c) Empleados(as)}

Existen diversos estudios que atribuyen a la falta de flexibilidad estructural en el trabajo, el estrés, la insatisfacción laboral, el bajo rendimiento y/o ausentismo de los empleados al tratar de combinar trabajo y familia. Algunos estudios en países desarrollados ${ }^{16}$ afirman que un gran número de empleados cambiarían algunos beneficios o compensaciones por medidas de flexibilidad.

En este orden de ideas,las orgnizaciones públicas y/o privadas--- en nuestro entorno-- en la medida que reconozcan el fenómeno sociodemografico gestado desde el ultimo tercio del siglo pasado de la incorporación masiva de la mujer al mundo laboral, de los roles compartidos en la condución del hogar,en el cuidado de los hijos y/o adultos mayores, y en solventar el gasto familiar; y sus efectos en el espacio Trabajo/Familia, deberán esforzarse unas y otras en diseñar :

1) políticas públicas complementarias a las derivadas del cumplimiento de obligaciones en materia laboral y de seguridad social, y

${ }^{16}$ Como los realizados por Families and Work Institute FWI,[online] disponible en :http://www.familiesandwork.org/national-study-for-the-changing-workforce/ 
2) políticas empresariales que favorezcan la armonía entre los espacios Trabajo/Familia.

3) Por su parte los empleados y empleadas también deberán contribuir a la observancia puntual de los compromisos adquiridos con la empresa y a proponer en su caso los cambios necesarios que interfieran o amenacen con interferir para su cumplimiento.

Lo anterior deberá producir efectos positivos en ambos lados, en el trabajo y en la familia de manera tal que se valore por parte del empleado(a) las políticas empresariales de flexibilidad laboral como una fuente de retribución laboral tan importante como la salarial en tanto que al empleador le facilitará el formar parte del grupo de empresas socialmente responsables coadyuvantes del gobierno en la consecución de los Objetivos del Milenio.

\section{Amanera de corolario}

Tanto las empresas como el gobierno y los empleados deben coadyuvar en encontrar la forma de armonizar el ámbito familiar y el laboral, pues de no hacerlo así, las empresas enfrentaran problemas serios en la productividad, rotación de personal, ausentismo y/o pérdida de empleados, los empleados observarán estrés, insatisfacción laboral, bajo rendimiento, ausentismo, renuncia y/o rotación; y el gobierno indicadores negativos en crecimiento económico, en generación de empleos, en niveles de salud, en calidad de vida, en bienestar y en alcanzar la igualdad sustantiva entre hombres y mujeres.

Si bien la Cuenta Satélite del Trabajo no Remunerado de los Hogares de México CSTNRHM, representa desde 2011, un avance sin precedentes en la producción de insumos para el diseño de políticas públicas dirigidas a la igualdad entre mujeres y hombres. Los datos arrojados en la CSTNRHM (2013) y en los años anteriores hacen visible las muchas prácticas discriminatorias hacia las mujeres trabajadoras y/o hacia las dedicadas exclusivamente a las labores del hogar, que aún imperan en el país, por la falta de valoración y reconocimiento de su 
participación en la economía del cuidado nacional por lo que urge institucionalizar en leyes laborales y fiscales las políticas públicas que promueven la armonización de los ámbitos laboral y familiar y buscan el objetivo de abatir las desigualdades que existen al interior del hogar y sus lamentables efectos sobre las mujeres.

\section{Bibliografía y Webgrafía}

Alderfer,Charleen,1994,A response to a feminist critique of organizational humanism,Journal of applied Behavioral Science,Vol.30

Families and Work Institute FWI,[online] disponible en :http://www.familiesandwork.org/national-study-for-the-changing-workforce/

INEGI,Conociendo México,2013[online]Disponible en : https://www.dane.gov.co/files/noticias/eventos/inegi_rmlg.pdf PIB y Cuentas Nacionales de México 2013.[online]Disponible en:http://www.inegi.org.mx/est/contenidos/proyectos/cn/tnrh/

Organización de las Naciones Unidas ONU Objetivos de desarrollo del Milenio, Conferencia Internacional sobre la Financiación para el Desarrollo celebrada en Monterrey, México, en 2002 y reafirmada en la Cumbre Mundial sobre Desarrollo Sostenible celebrada en Johannesburgo en agosto de 2002. ONU México,[online] Disponible en: http://www.onu.org.mx/objetivos_de_desarrollo_del_milenio.html ${ }^{\wedge}$ consultado el $12 / 05 / 2015$ 